\title{
Osteoclast-related cytokines from biopsy specimens predict mandibular invasion by oral squamous cell carcinoma
}

\author{
NIANHUI CUI ${ }^{1}$, TAKESHI NOMURA ${ }^{2}$, NOBUO TAKANO ${ }^{2}$, ENBO WANG $^{1}$, \\ WEI ZHANG $^{1}$, TAKESHI ONDA ${ }^{2}$ and TAKAHIKO SHIBAHARA ${ }^{2}$ \\ ${ }^{1}$ Department of Oral and Maxillofacial Surgery, Peking University School and Hospital of Stomatology, Beijing, \\ P.R. China; ${ }^{2}$ Department of Oral and Maxillofacial Surgery, Tokyo Dental College, Chiba, Japan
}

Received May 20, 2010; Accepted July 13, 2010

DOI: $10.3892 /$ etm.2010.128

\begin{abstract}
The aim of this study was to examine the value of osteoclast-related cytokines in biopsy specimens for predicting mandibular invasion by oral squamous cell carcinoma (OSCC). In this retrospective study, biopsy specimens were obtained from 30 patients with OSCC. We observed the expression of seven osteoclast-related cytokines (IL-1 $\alpha$, RANKL, PTHrP, OPG, IL-6, RANK and TNF- $\alpha$ ) using immunohistochemical (IHC) staining and of an osteoclast using tartrate-resistant acid phosphatase (TRAP) staining. The results were as follows: i) double-staining and IHC staining for RANKL, PTHrP and IL- $1 \alpha$ in biopsy samples had diagnostic potential for predicting mandibular invasion; ii) TRAP-positive monoor multinuclear cells were noted in the biopsy samples; iii) double-positive or -negative findings appeared to reliable indicate whether samples were invasion-positive or invasionnegative. Positive IHC staining for PTHrP, IL-1 $\alpha$ or RANKL appeared to typically indicate an invasion-positive lesion. We suggest that the expression of both osteoclasts and osteoclastrelated cytokines can be used to predict mandibular invasion.
\end{abstract}

\section{Introduction}

Mandibular invasion of oral squamous cell carcinoma (OSCC) is a significant problem for oral surgeons. Such invasion has a direct impact on surgical strategies and patient prognosis (1). Although the final diagnosis must be made through postoperative pathological examination, mandibular invasion is most commonly suspected from pre-operative radiography or computed tomography $(2,3)$. In clinical work, making a decision based on imaging alone is sometimes difficult

Correspondence to: Dr Takahiko Shibahara, Department of Oral and Maxillofacial Surgery, Tokyo Dental College, 1-2-2 Masago Mihamaku, Chiba 261-8502, Japan

E-mail: sibahara@tdc.ac.jp

Key words: oral squamous cell carcinoma, mandibular invasion, osteoclast-related cytokine, immunohistochemistry, tartrate-resistant acid phosphatase staining
$(4,5)$. Alternative methods for the pre-operative detection of mandibular invasion are thus required.

During bone invasion, osteoclasts still represent the terminal cells for the absorption of bone. With malignant lesions, osteoclasts are stimulated and activated through general mechanisms. Certain cytokines, such as receptor activator of nuclear factor- $\kappa \mathrm{B}$ (RANK) ligand (RANKL), tumor necrosis factor (TNF)- $\alpha$, interleukin (IL)-1 $\alpha$ and -6 , parathyroid hormone-related protein (PTHrP) and osteoprotegerin (OPG) are known to participate in this process (6-8). Most research in this area has focused on tissues adjacent to the bone. Biopsy samples have not commonly been used due to their distance from bone. However, OSCC with the ability to invade bone would presumably display telltale biological characteristics even in superficial regions of the tumor from which the biopsy is taken. Based on our previous research (9), this study examined the value of osteoclast-related cytokines in biopsy specimens for predicting mandibular invasion by OSCC using both immunohistochemical (IHC) and tartrateresistant acid phosphatase (TRAP) staining.

\section{Materials and methods}

Patients and specimens. In this retrospective study, biopsy specimens (when available) were obtained from 30 patients with OSCC. Original tumor locations were in the lower gingiva or oral floor. Background data for patients are shown in Table I. Diagnoses of OSCC and mandible invasion were based on serial slices stained with H\&E $(10,11)$. Paraffin block biopsy specimens of these 30 patients were retrieved and sliced into sections at a thickness of $4 \mu \mathrm{m}$. All slices were inspected using digital images captured using Axioplan2 and Axiophot2 microscopes (Carl Zeiss, Jena, Germany).

Immunohistochemical staining of osteoclast-related cytokines. Seven antibodies against osteoclast-related cytokines were used in this study: anti-IL-1 $\alpha$ (Endogen, Woburn, MA, USA), anti-IL-6 (Santa Cruz Biotechnology, Santa Cruz, CA, USA), anti-OPG (R\&D Systems, Minneapolis, MN, USA), anti-PTHrP (EMD Chemicals, San Diego, CA, USA), anti-RANK (R\&D Systems), anti-RANKL (R\&D Systems), and anti-TNF- $\alpha$ (goat anti-human polyclonal antibody; R\&D Systems). 
Table I. General characteristics of the patients.

\begin{tabular}{lr}
\hline Gender & \\
Male & 21 \\
Female & 9 \\
Age (years) & \\
Range & $36-85$ \\
Average & 64 \\
T stage & \\
1 & 1 \\
2 & 10 \\
3 & 6 \\
4 & 13 \\
N stage & \\
0 & 9 \\
1 & 11 \\
2 & 10 \\
M (0) & 30 \\
Bone invasion & \\
Positive & 14 \\
Negative & 16 \\
\hline
\end{tabular}

Immunohistochemical analysis was performed using the Streptomyces anti-biotin protein-peroxidase (SP) method with a Histofine SAB-PO kit (Nichirei, Tokyo, Japan). Sections were deparaffinized in xylene and rehydrated by serial incubation in a graded ethanol series followed by washign in phosphate-buffered saline (PBS). Antigen retrieval was performed by microwave heating in $0.01 \mathrm{M}$ citrate buffer at $95^{\circ} \mathrm{C}$ for $5 \mathrm{~min}$. Endogenous peroxidase activity was quenched by the addition of $3 \%$ hydrogen peroxide in methanol for $15 \mathrm{~min}$. After washing in PBS, sections were incubated in normal rabbit serum for $30 \mathrm{~min}$ at room temperature to prevent non-specific protein binding, then incubated with primary antibodies diluted 1:100 in a humidified chamber at $37^{\circ} \mathrm{C}$ for $1 \mathrm{~h}$. Sequential incubations were then performed with biotin-labeled secondary antibodies and peroxidaselabeled streptavidin at $37^{\circ} \mathrm{C}$ for $30 \mathrm{~min}$, in accordance with the instructions from the reagent kit. The sections were washed thoroughly in PBS between two incubations. A duration of 1-5 min was required to reveal brown products with Histofine Simplestain DAB solution (Nichirei) under a microscope. A section with PBS instead of the primary antibodies was used as the negative control in every case. Slight counterstaining with hematoxylin was performed as a final step.

Tartrate-resistant acid phosphatase staining. The dewaxing and rehydrating procedures were the same as those used in IHC staining. After washing with PBS, a TRAP kit (Cell Garage, Tokyo, Japan) was used for TRAP staining. Incubation lasted for 30-60 min at $37^{\circ} \mathrm{C}$ and was monitored under a microscope. Hematoxylin was used to counterstain the cell nucleus.

Double-staining with IHC and TRAP staining. Doublestainings were performed to clarify the relationships between TRAP-positive cells, osteoclasts and pre-osteoclasts, and cytokine-positive cells upon IHC staining. TRAP staining was performed just before antigen unmasking. Subsequently, after washing with PBS, IHC staining was continued following the above-mentioned protocol. Sections were dipped into hematoxylin for the light conterstaining of nuclei.

Evaluation of positive-stained tissues. Sections were reviewed independently with $\mathrm{H} \& \mathrm{E}$ staining after IHC and TRAP staining. The characteristics and locations of positive cells were recorded, and the rates were calculated for positive $\mathrm{H} \& \mathrm{E}$ staining for each cytokine and for TRAP staining, excluding the OPG cytokine. Positive rates of IHC staining, TRAP staining and double-staining were compared to histopathological diagnoses for uniformity. The diagnostic accuracy of the staining for each cytokine and double-staining compared to $H \& E$ staining was then determined according to a formula for sensitivity and specificit. Positive and negative predictive values of the dye applications were then calculated. A significant correlation between the expression of these osteoclast-related cytokines and mandibular invasion status was evaluated by the Fisher's exact test. A value of $\mathrm{P}<0.05$ indicated a significant difference.

\section{Results}

Staining and statistical results in gingival or oral floor OSCC. IHC staining revealed positive-stained cells in the specimens as follows: IL-1 $\alpha$, lymphocytes; RANKL, stromal cell-like cells and lymphocytes; PTHrP, tumor cells; OPG, stromal cell-like cells; IL-6, lymphocytes; RANK, osteoclast-like cells; and TNF- $\alpha$, tumor cells and lymphocytes. Fig. 1 illustrates the positive staining for the expression of RANKL and IL-1 $\alpha$. Fig. 2 shows that the positive results for TRAP staining included monocytes, pre-osteoclast-like and osteoclastlike cells. Some TRAP-positive cells were observed in the epithelium of capillaries in the tumor tissues. Fig. 3 shows the positive results for IHC and TRAP staining. The antibody of the cytokine was IL- $1 \alpha$. It was possible to distinguish between ICH-positive and TRAP-positive cells.

Diagnostic accuracy of cytokine IHC staining. Positive rates of IHC staining in bone invasive OSCC were: IL-1 $\alpha, 62.5 \%$; IL-6, 37.5\%; OPG, 90\%; RANK, 80\%; RANKL, 83.3\%; PTHrP, $88.9 \%$ and TNF- $\alpha, 55.6 \%$. The positive predictive value of IL-1 $\alpha$, IL-6, RANK, PTHrP was higher than that of the other cytokines, and the negative predictive value of IL-1 $\alpha$, OPG, RANK, RANKL, PTHrP was higher than that of the other cytokines. IL-1 $\alpha$, RANK and PTHrP were shown to have better uniformity than the other cytokines (Table II). There was significant correlation between the expression of IL-1 $\alpha$, OPG, RANKL, PTHrP and mandibular invasion status $(\mathrm{P}<0.05)$. While most OSCC samples with positive staining of IL-1 $\alpha$, RANKL and PTHrP had bone invasion, tumors without invasive lesions were negative for these osteoclast-related cytokines. In contrast, most of the OPG-negative-stained cases significantly exhibited bone invasion.

Diagnostic accuracy of double-staining using IHC and TRAP staining. All double-positive tissues for IHC and TRAP staining were H\&E invasion-positive, and all double-negative tissues were H\&E invasion-negative. The diagnostic accuracy 

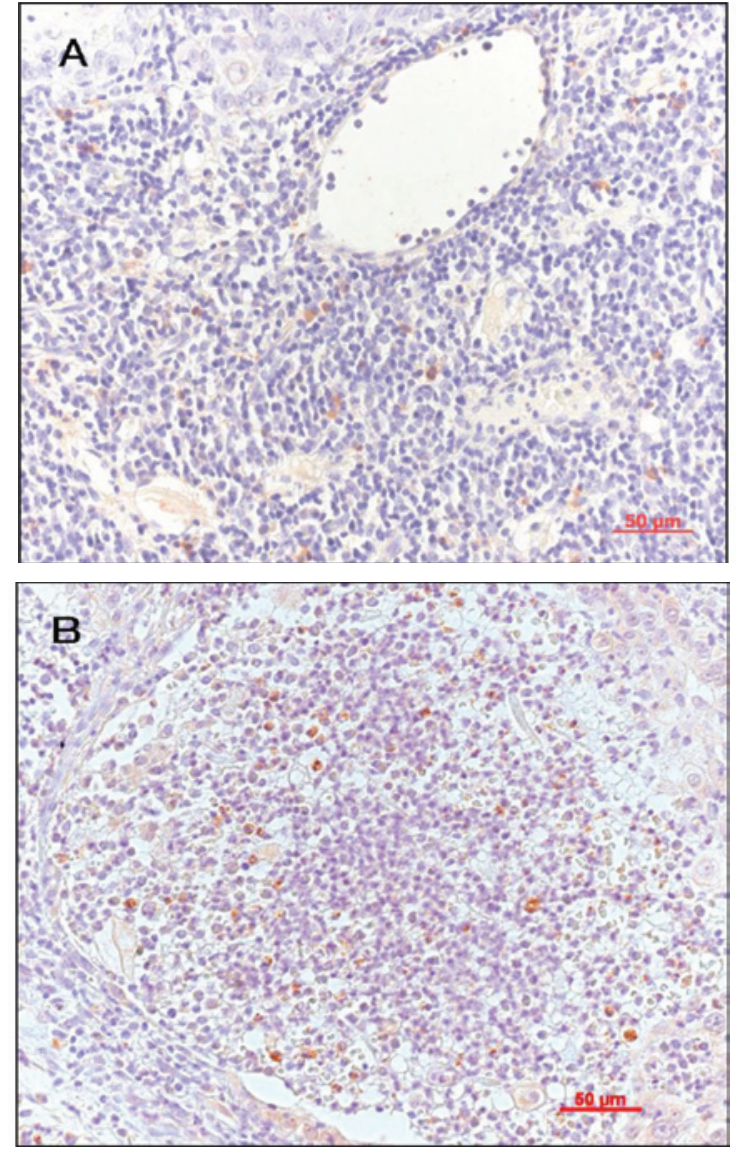

Figure 1. IHC staining of osteoclast-related cytokines. (A) Positive staining for RANKL: stromal cell-like cells and lymphocytes (x200). (B) Positive staining for IL-1 $\alpha$ : lymphocytes (x200).

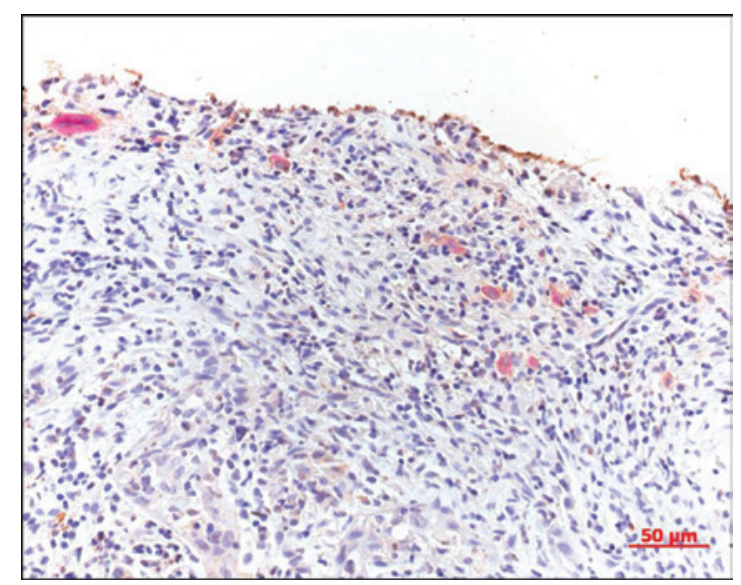

Figure 2. Positive TRAP staining. Monocytes, pre-osteoclast-like cells and osteoclast-like cells (x200).

was a complete match for the positive tissues concerning the expression of each of the cytokines and TRAP (Table III).

\section{Discussion}

The more information obtained pre-operatively regarding the patient and disease, the better the outlook is for treatment and prognosis. In cases of OSCC, determining whether mandibular
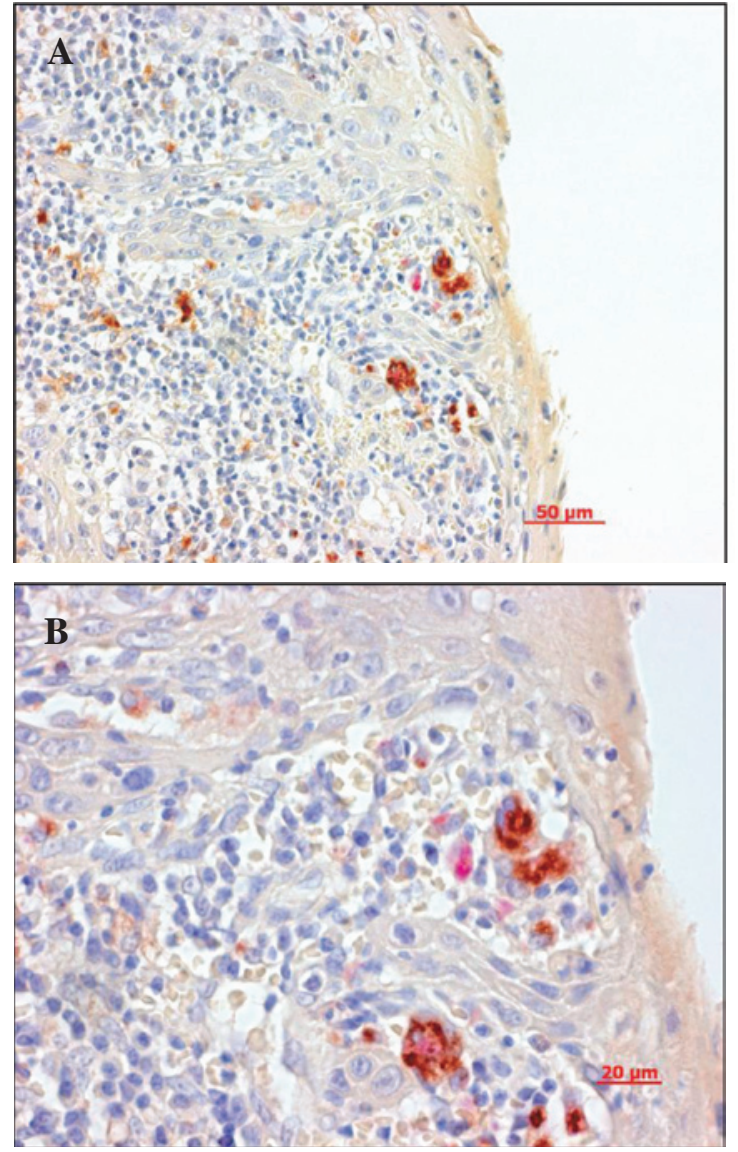

Figure 3. Double-staining for IHC and TRAP. The antibody was for IL-1 $\alpha$. Cytokine-negative cells were so close to TRAP-positive cells that some cells were stained in two colors (A, x200; B, x400).

invasion has occurred is critical for the oral surgeon. Clinical features and medical imaging modalities, such as radiography, computed tomography and magnetic resonance imaging, are commonly used in this situation. However, the sensitivity and specificity of these systems remain problematic $(2,4)$. Ideally, determination of mandibular invasion should be performed using a highly sensitive and specific test that is both inexpensive and non-invasive.

Pre-operative biopsy indicates certain characteristics of the tumor. However, in cases of OSCC with potential mandibular invasion, estimating invasion based solely on an $\mathrm{H} \& \mathrm{E}$-stained biopsy specimen is difficult, since the interface between the carcinoma and bone is not directly distinguishable (10). Research to determine whether osteoclast-related cytokines show relationships between carcinoma and bone has revealed that some cytokines play important roles in the mandibular invasion of OSCC (6). We therefore aimed to ascertain whether these cytokines in biopsy specimens can predict bone invasion.

Many cytokines participate in osteoclast differentiation and activation. RANKL, RANK and OPG are regarded as the basic cytokines influencing the entire process. Other cytokines, such as TNF- $\alpha$, IL-1 $\alpha$ and PTHrP, have also been found to exert certain effects on osteoclasts $(12,13)$. The present study found that all of the investigated 7 cytokines in the biopsy samples of gingival OSCC indicated mandibular invasion. 
Table II. The diagnostic accuracy of the cytokines.

\begin{tabular}{|c|c|c|c|c|c|c|c|c|}
\hline & \multicolumn{3}{|c|}{$\begin{array}{l}\text { Bone invasion } \\
\text { (by } H \& E \text { ) }\end{array}$} & \multirow[t]{2}{*}{ P-value } & \multirow[t]{2}{*}{ Specificity } & \multirow[t]{2}{*}{ Sensitivity } & \multirow[t]{2}{*}{$\begin{array}{l}\text { Positive predictive } \\
\text { value }\end{array}$} & \multirow[t]{2}{*}{$\begin{array}{l}\text { Negative predictive } \\
\text { value }\end{array}$} \\
\hline & + & - & Total & & & & & \\
\hline \multicolumn{9}{|c|}{ IL- $1 \alpha$} \\
\hline+ & 5 & 0 & 5 & & & & & \\
\hline \multirow[t]{2}{*}{-} & 3 & 11 & 14 & 0.0048 & 1 & 0.63 & 1 & 0.79 \\
\hline & 8 & 11 & 19 & & & & & \\
\hline \multicolumn{9}{|c|}{ IL-6 } \\
\hline+ & 3 & 1 & 4 & & & & & \\
\hline \multirow[t]{2}{*}{-} & 5 & 9 & 14 & 0.2745 & 0.90 & 0.38 & 0.75 & 0.64 \\
\hline & 8 & 10 & 18 & & & & & \\
\hline \multicolumn{9}{|c|}{$\mathrm{OPG}$} \\
\hline+ & 9 & 5 & 14 & & & & & \\
\hline \multirow[t]{2}{*}{-} & 1 & 13 & 14 & 0.0044 & 0.72 & 0.90 & 0.64 & 0.93 \\
\hline & 10 & 18 & 28 & & & & & \\
\hline \multicolumn{9}{|c|}{ RANK } \\
\hline+ & 4 & 1 & 5 & & & & & \\
\hline \multirow[t]{2}{*}{ - } & 1 & 6 & 7 & 0.0720 & 0.86 & 0.8 & 0.80 & 0.86 \\
\hline & 5 & 7 & 12 & & & & & \\
\hline \multicolumn{9}{|c|}{ RANKL } \\
\hline+ & 10 & 7 & 17 & & & & & \\
\hline \multirow[t]{2}{*}{ - } & 2 & 11 & 13 & 0.0256 & 0.61 & 0.83 & 0.59 & 0.85 \\
\hline & 12 & 18 & 30 & & & & & \\
\hline \multicolumn{9}{|c|}{ PTHrP } \\
\hline+ & 8 & 1 & 9 & & & & & \\
\hline \multirow[t]{2}{*}{-} & 1 & 10 & 11 & 0.0009 & 0.91 & 0.89 & 0.89 & 0.91 \\
\hline & 9 & 11 & 20 & & & & & \\
\hline \multicolumn{9}{|c|}{ TNF- $\alpha$} \\
\hline+ & 5 & 2 & 7 & & & & & \\
\hline \multirow[t]{2}{*}{-} & 4 & 9 & 13 & 0.1597 & 0.82 & 0.56 & 0.71 & 0.69 \\
\hline & 9 & 11 & 20 & & & & & \\
\hline
\end{tabular}

${ }^{\mathrm{a} B y}$ Fisher's exact test. Values in bold are significant.

In the TRAP staining, the positive cells consisted of monocytes, pre-osteoclasts and osteoclasts. These cells are believed to be in the early stages of osteoclast maturation (14). In carcinomas with the potential for bone invasion, tumor cells express factors that allow further maturation of pre-osteoclasts. When this does not occur, the pre-osteoclasts remain in an early stage. This may be why the TRAP-positive cells were observed in both the invasion-positive and invasion-negative specimens. However, only tumor tissues with the ability to express sufficient osteoclast-related cytokines promote preosteoclasts and osteoclasts to differentiate and activate.

Based on the percentage of positive cells for each cytokine, we hypothesized a network of tumor cells, osteoclasts and other cells connected with cytokines (Fig. 4). Bone-invasive tumor cells expressed TNF- $\alpha$, IL- $1 \alpha$ and PTHrP. These cytokines have been found to influence lymphocytes and endothelial cells to induce monocyte migration from blood vessels and differentiation into pre-osteoclasts via RANKL, which is expressed by capillary endothelial cells in the tumor
(15). Stromal cells and osteoblasts, which are also influenced by tumor-expressed cytokines, regulate the differentiation and activation of pre-osteoclasts and osteoclasts via RANKL and OPG.

Statistical analysis of the diagnostic uniformity of the bone invasion according to $\mathrm{H} \& \mathrm{E}$-stained sections revealed significant correlations with invasiveness for RANKL, IL-1 $\alpha$, PTHrP and OPG. Ranked in descending order according to diagnostic accuracy, these four cytokines were PTHrP, IL-1 $\alpha$, OPG and RANKL. Based on these results, negative staining for OPG was used due to the negative correlation with bone invasion. However, to exclude the presence of the antibody based solely on negative results from IHC staining was not possible, therefore a negative finding for OPG was considered unsuitable for diagnosis. The remaining three cytokines, PTHrP, IL-1 $\alpha$ and RANKL, were closly associated with bone invasion and demonstrated diagnostic potential.

In both physical and pathological situations, bone resorption is performed by osteoclasts. The number, the differentiation 
Table III. The diagnostic accuracy of the double staining.

\begin{tabular}{|c|c|c|c|c|}
\hline & \multicolumn{3}{|c|}{ Bone invasion $^{a}$} & \multirow[t]{2}{*}{ P-value } \\
\hline & + & - & Total & \\
\hline \multicolumn{5}{|c|}{ IL-1 $\alpha+$ TRAP } \\
\hline+ & 5 & 0 & 5 & \multirow{3}{*}{0.0005} \\
\hline \multirow[t]{2}{*}{-} & 0 & 9 & 9 & \\
\hline & 5 & 9 & 14 & \\
\hline \multicolumn{5}{|c|}{ IL-6 + TRAP } \\
\hline+ & 3 & 0 & 3 & \multirow{3}{*}{0.0083} \\
\hline \multirow[t]{2}{*}{-} & 0 & 7 & 7 & \\
\hline & 8 & 10 & 18 & \\
\hline \multicolumn{5}{|c|}{$\mathrm{OPG}+\mathrm{TRAP}$} \\
\hline+ & 10 & 0 & 10 & \multirow{3}{*}{$<0.0001$} \\
\hline \multirow[t]{2}{*}{-} & 0 & 12 & 12 & \\
\hline & 10 & 12 & 22 & \\
\hline \multicolumn{5}{|c|}{ RANK + TRAP } \\
\hline+ & 3 & 0 & 3 & \multirow{3}{*}{0.0250} \\
\hline- & 0 & 1 & 1 & \\
\hline & 3 & 1 & 4 & \\
\hline \multicolumn{5}{|c|}{ RANKL + TRAP } \\
\hline+ & 10 & 0 & 10 & \multirow{3}{*}{$<0.000$} \\
\hline- & 0 & 10 & 10 & \\
\hline & 10 & 10 & 20 & \\
\hline \multicolumn{5}{|c|}{ PTHrP + TRAP } \\
\hline+ & 8 & 0 & 8 & \multirow{3}{*}{0.0002} \\
\hline- & 0 & 8 & 8 & \\
\hline & 8 & 8 & 16 & \\
\hline \multicolumn{5}{|c|}{ TNF- $\alpha+$ TRAP } \\
\hline+ & 5 & 0 & 5 & \multirow{3}{*}{0.0013} \\
\hline \multirow[t]{2}{*}{-} & 0 & 7 & 7 & \\
\hline & 5 & 7 & 12 & \\
\hline
\end{tabular}

aBy H\&E; by Fisher's exact test.

and the activation of osteoclasts are directly related to the outcome of bone resorption. Cytokines regulating osteoclast activity thus participate in this process. IHC and TRAP staining was carried out together in one section. The location and relationship of the cytokine-expressing and osteoclast-like cells were revealed more clearly and directly in this investigation. Cytokine-negative cells were so close to TRAP-positive cells that some cells exhbited staining in two colors, and cytokines, such as RANKL, IL-1 $\alpha$ and PTHrP, were confirmed to be strong regulators of mandibular invasion by OSCC. In cases of OSCC showing bone invasion, TRAP-positive osteoclasts were previously identified at the interface between tumor and bone (9). In the present study, TRAP-positive cells, pre-osteoclasts and osteoclast-like cells were noted in the biopsy tissues only in specimens with bone invasion, and not in invasion-negative specimens. Some TRAP-positive cells were found in the walls of tumor capillaries, indicating that certain pre-osteoclasts may have originated from the circulation. Such cells would be induced by various factors in cancer tissue to pass through the

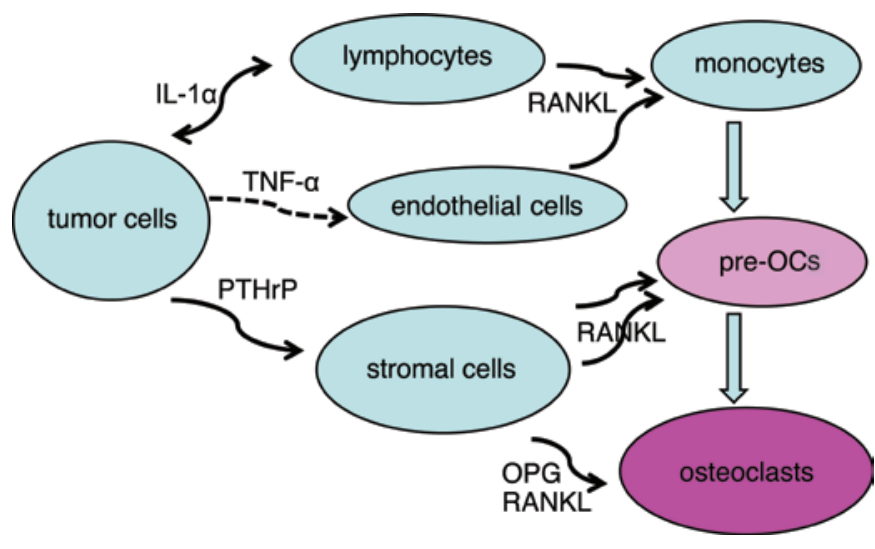

Figure 4. Network of tumor cells and osteoclasts connected with cytokines.

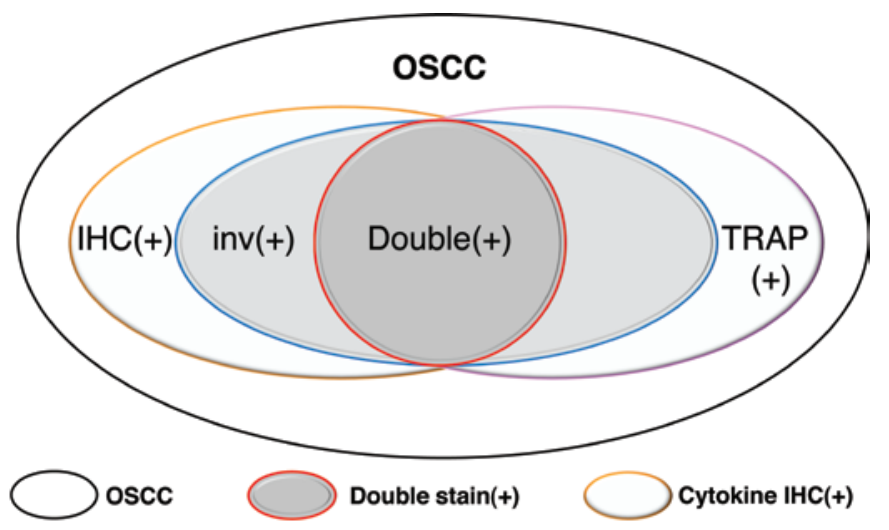

H\&E bone invasion(+)

$\operatorname{TRAP}(+)$

Figure 5. Relationships between mandibular invasion and IHC, TRAP staining and double-staining in OSCC cases.

capillary endothelium, where they then activate and differentiate into functional osteoclasts.

In cytokine IHC and TRAP double-staining, when the result was double-positive or double-negative, the sensitivity and specificity were $100 \%$. This means that double-staining offers high diagnostic value for mandibular invasion by OSCC. However, samples showing single-positive results sometimes indicated bone invasion and sometimes not. Thus, the use of double-staining as a diagnostic criterion resulted in some false-negatives, but no false-positives (Fig. 5).

As previously mentioned, IHC and TRAP staining of biopsy specimens offered a certain diagnostic value for the prediction of mandibular invasion by OSCC. This may be divided into three levels of certainty: i) double-positive or double-negative, definite diagnosis of invasion or non-invasion; ii) positive IHC staining for PTHrP, IL-1 $\alpha$ or RANKL, most of the specimens represent bone invasion; iii) positive IHC staining for other cytokines or TRAP staining only, combining clinical features and medical imaging would offer a better indication of possible bone invasion.

Originally, gingiva is placed in an inflammatory milieu. It is a non-specific environment which is common in all adults, in particular the elderly. However, the gingival environment in the case of gingival cancer with bone invasion may be 
considered a specific environment or a 'further exacerbation of inflammation'. In other words, it is assumed to present the 'further increased expression of osteoclastic cytokines'. In this study, we evaluated the exacerbation of the inflammatory reaction in gingival cancer with bone invasion by scoring IHC staining, using gingival cancer without bone invasion as the control. A stronger expression of inflammatory cytokines (osteoclastic cell-induced cytokines) and osteoclastic cells was noted in the bone invasion group. The stronger inflammatory reaction was not caused simply by the gingival cancer, but by the presence of bone invasion. This was considered to be a specific inflammatory reaction, which was not observed in specimens of ordinary gingiva.

The establishment of novel screening techniques for the detection of mandibular invasion before treatment is needed. OSCC, which has the potential for mandibular invasion, demonstrates more invasive biological characteristics. Whether such invasive potential is associated with metastatic potential represents a question for future study (16). In conclusion, the expression of both osteoclasts and osteoclast-related cytokines is an important factor for the prediction of mandibular invasion.

\section{Acknowledgements}

This study was supported by a research grant from the Ministry of Education, Science and Culture, Japan.

\section{References}

1. Genden EM, Rinaldo A, Jacobson A, Shaha AR, Suarez C, Lowry J, Urguhart AC, Werner JA, Gullane PJ and Ferlito A: Management of mandibular invasion: when is a marginal mandibulectomy appropriate? Oral Oncol 41: 776-782, 2005.

2. Nakayama E, Yoshiura K, Ozeki S, Nakayama H, Yamaguchi T, Yoshikawa H, Kanda S, Ohishi M and Shirasuna K: The correlation of histological features with a panoramic radiography pattern and a computed tomography pattern of bone destruction in carcinoma of the mandibular gingival. Oral Surg Oral Med Oral Radiol Endod 96: 774-782, 2003.

3. Brockenbrough JM, Petruzzelli GJ and Lomasney L: Dentascan as an accurate method of predicting mandibular invasion in patients with squamous cell carcinoma of oral cavity. Arch Otolaryngol Head Neck Surg 129: 113-117, 2003.
4. Imaizumi A, Yoshino N, Yamada I, Nagumo K, Amagasa T, Omura K, Okada N and Kurabayashi T: A potential pitfall of MR imaging for assessing madibular invasion of squamous cell carcinoma in oral cavity. Am J Neuroradiol 27: 114-122, 2006.

5. Ash CS, Nason RW, Abdoh AA and Cohen M: Prognostic implication of mandibular invasion in oral cancer. Head Neck 22: 794-798, 2000

6. Shibahara T, Nomura T, Cui NH and Noma H: A study of osteoclast-related cytokines in mandibular invasion by squamous cell carcinoma. Int J Oral Maxillofac Surg 34: 789-793, 2005.

7. Tada T, Jimi E, Okamoto M, Ozeki S and Okabe K: Oral squamous cell carcinoma cells induce osteoclast differentiation by suppression of osteoprotegerin expression in osteoblasts. Int J Cancer 116: 253-262, 2005.

8. Dunne FP, Bowden SJ, Brown JS, Ratcliffe WA and Browne RM: Parathyroid hormone related protein in oral squamous cell carcinomas invading the mandible. J Clin Pathol 48: 300-303, 1995.

9. Cui N, Nomura T, Noma H, Yokoo K, Takagi R, Hashimoto S, Okamoto M, Sato M, Yu G, Guo C and Shibahara T: Effect of YM529 on a model of mandibular invasion by oral squamous cell carcinoma in mice. Clin Cancer Res 11: 2713-2719, 2005.

10. Lukinmaa PL, Hietanen J, Söderholm AL and Lindgvis C: The histologic pattern of bone invasion by squamous cell carcinoma of the mandibular region. Br J Oral Maxillofac Surg 30: 2-7, 1992.

11. Brown JS, Lowe D, Kalavrezos N, D'Souza J, Magennis P and Woolgar J: Patterns of invasion and routes of tumor entry into the mandible by oral squamous cell carcinoma. Head Neck 24: 370-383, 2002.

12. Azuma Y, Kaji K, Katogi R, Takeshita S and Kudo A: Tumor necrosis factor-alpha induces differentiation of and bone resorption by osteoclasts. J Biol Chem 275: 4858-4864, 2000.

13. Kobayashi K, Takahashi N, Jimi E, Udagawa N, Takami M, Kotake S, Nakagawa N, Kinosaki M, Yamaguchi K, Shima N, Yasuda H, Morinaga T, Higashio K, Martin TJ and Suda T: Tumor necrosis factor alpha stimulates osteoclast differentiation by a mechanism independent of the ODF/RANKL-RANK interaction. J Exp Med 191: 275-286, 2000.

14. Van de Wijingaert FP and Burger EH: Demonstration of tartrate-resistant acid phosphatase in un-decalcified, glycolmethacrylate-embedded mouse bone: a possible marker for (pre) osteoclast identification. J Histochem Cytochem 34: 1317-1323, 1986.

15. Cui NH, Zhang W, Wang EB, Wei MJ, Guo CB and Yu GY: Osteoclastic bone destruction and its regulating factors in oral squamous cell carcinoma. Beijing Da Xue Xue Bao 39: 30-32, 2007.

16. Jones DH, Nakashima T, Sanchez OH, Kozieradzki I, Komarova SV, Sarosi I, Morony S, Rubin E, Sarao R, Hojilla CV, Komnenovic V, Kong YY, Schreiber M, Dixon SJ, Sims SM, Khokha R, Wada T and Penninger JM: Regulation of cancer cell migration and bone metastasis by RANKL. Nature 440: 692-696, 2006 